\title{
Basic Fibroblast Growth Factor Enhances the Coupling of Intimal Hyperplasia and Proliferation of Vasa Vasorum in Injured Rat Arteries
}

\author{
Elazer R. Edelman, ${ }^{\star *}$ Matthew A. Nugent, ${ }^{\star}$ Laura T. Smith, and Morris J. Karnovsky \\ *Cardiovascular Division, Department of Internal Medicine, Brigham and Women's Hospital and Harvard Medical School, \\ Boston, Massachusetts 02115; ${ }^{\ddagger}$ Harvard-Massachusetts Institute of Technology, Division of Health Sciences and Technology, \\ Massachusetts Institute of Technology, Cambridge, Massachusetts 02139; and ${ }^{\S}$ Department of Pathology, \\ Harvard Medical School, Boston, Massachusetts 02115
}

\begin{abstract}
Basic fibroblast growth factor (bFGF) is mitogenic for smooth muscle cells (SMC) and angiogenic. We examined the in vivo effects of bFGF in balloon denuded carotid arteries of laboratory rats. bFGF was administered continuously from polymerbased devices at $34 \mathrm{ng} / \mathrm{d}$ into the periadventitial space of rat carotid arteries for $\mathbf{2}$ wk. Intimal hyperplasia was not observed in the absence of injury or with lipopolysaccharide induced endothelial dysfunction. Different degrees of vascular injury produced proportionally more intimal hyperplasia. bFGF increased the intimal hyperplastic response 1.3-fold with severe vascular injury, and 2.4-fold with more mild injury. Increased cell proliferation, not extracellular matrix production, accounted for these effects. Cell density was unchanged for the control and bFGF-treated groups, and the number of proliferating intimal cells at 2 wk rose to an amount equivalent to the increase in mass; 1.9- and 4.0-fold for severe and lesser injury, respectively. The relative ability of heparin to reduce SMC proliferation was not altered by the presence of bFGF. bFGF also induced profound angiogenesis within and surrounding the polymeric releasing device, and in the vasa vasorum immediately around the injured arteries. bFGF's effect on vasa was linearly related to the amount of SMC proliferation within the blood vessel. Thus, the in vivo mitogenic and angiogenic potential of bFGF are coupled, and may be similarly modulated by the products of local injury and/or factors in the vessel wall. ( $J$. Clin. Invest. 1992. 89:465-473.) Key words: arterial injury • controlled drug delivery • heparin • lipopolysaccharide • restenosis • smooth muscle cell proliferation
\end{abstract}

\section{Introduction}

Control of the intimal response to vascular injury has been the focus of much recent research. Accelerated atherosclerosis is increasingly being recognized as an important rate limiting factor in angioplasty, vascular bypass surgery and organ transplantation $(1,2)$. We have focused on two aspects of this phenomenon; the neointimal proliferation of smooth muscle cells

Address reprint requests to Dr. Edelman, Department of Pathology, Building D-2, Room 336, Harvard Medical School, 200 Longwood Avenue, Boston, MA 02115.

Received for publication 21 March 1991 and in revised form 17 September 1991.

J. Clin. Invest.

(C) The American Society for Clinical Investigation, Inc.

0021-9738/92/02/0465/09 \$2.00

Volume 89, February 1992, 465-473
$(\mathrm{SMC})^{1}$ and the perivascular proliferation of microvasculature or vasa vasorum. ${ }^{2} \mathrm{~A}$ variety of agents have been suggested as potential modulators of SMC proliferation including heparin (3); immunosuppressants such as cyclosporine (4), calcium channel blockers $(5,6)$, captopril, and other angiotensin-converting enzyme inhibitors (7); and growth factors and their inhibitors (8). Heparin, for example, reduced the proliferation of SMC in tissue culture at concentrations as low as $10 \mu \mathrm{g} / \mathrm{ml}(9)$, and in a number of arterial systems following mechanical injury $(3,10)$. The perivascular administration of heparin, and a modified nonanticoagulant heparin derivative, resulted in local concentrations of the drug without systemic dosing, and proved to be as effective as the intravenous administration of the same compounds (11).

The area around blood vessels is rich in vasa vasorum, especially in the vicinity of atherosclerotic lesions (12-15). In order to investigate the possibility that the vasa and the neointimal responses are coupled or mutually regulated we examined the effects of the periadventitial delivery of an agent that is both mitogenic for SMCs and angiogenic. Basic fibroblast growth factor (bFGF) $)^{1}$ is synthesized by endothelial cells $(16,17)$, macrophages, and SMCs (18) and is mitogenic for endothelial cells and SMCs (19-21), and angiogenic (19, 22-25). It is associated with the extracellular matrix and basement membrane (2629). For these and other reasons it has been postulated that bFGF may play an important role in the pathogenesis of atherosclerotic vascular disease (30). Lindner and co-workers (31) localized bFGF within the normal rat aorta, demonstrated that rat carotid artery SMC express the mRNA for bFGF, and showed that though SMC proliferation soon after arterial injury was diminished by antibodies to bFGF the ultimate size of the neointimal lesion was left unaffected (32). Systemic administration of bFGF enhanced endothelial replication and overgrowth 8 wk after denuding injury (33), and increased SMC proliferation but only in injured carotid arteries (31). They concluded that "the endothelium may represent an effective barrier for this mitogen and therefore the smooth muscle cells were never exposed to effective concentrations of bFGF."

In this study we have measured the effects of bFGF on the vascular response to injury when the growth factor was applied to the perivascular region of rat carotid arteries. This enabled us to deliver bFGF to medial SMC without removing the endothelial "barrier" and to ask whether there might be some other

1. Abbreviations used in this paper: bFGF, basic fibroblast growth factor; EVAc, ethylene-vinyl acetate copolymer; I/M, intimal/medial area ratio; PCNA, proliferating cell nuclear antigen; SMC, smooth muscle cell.

2. We defined vasa vasorum as those vessels residing within a zone one arterial width outward from the adventitia (see Fig. 1). 
function of the intact endothelium that could account for its inhibition of bFGF activity. We also extended the examination of the effects of bFGF to include local angiogenesis, as well as intimal hyperplasia and SMC proliferation. Neither intimal hyperplasia nor SMC proliferation was observed with bFGF in the absence of endothelial denuding vascular injury. In injured arteries bFGF induced an increase in neointimal SMC proliferation and an increase in the microvasculature surrounding the artery. Moreover, there was close correlation between the extent to which bFGF enhanced intimal hyperplasia, and the degree of angiogenesis induced by the growth factor. Thus, it appears that the degree of bFGF-stimulated neointimal proliferation after arterial injury is linked with bFGF neovascularization of the injured blood vessel.

\section{Methods}

Materials. A 2 French Fogarty catheter (American Edwards Laboratories, Santa Ana, CA) was used to induce endothelial injury in male Sprague-Dawley rats (300-500 g; Charles River Breeding Laboratories, Kingston, MA). Heparin-Sepharose beads were from Pharmacia LKB Biotechnology Inc., Piscataway, NJ, and purified human recombinant bFGF corresponding to the 146-amino acid form of the growth factor $(30,34,35)$ was from Chiron Inc., Emeryville, CA. Iodinated bFGF was prepared using a modification (34) of the method of Bolton and Hunter with specific radioactivity ranging from 25 to $100 \mathrm{nCi} / \mathrm{ng}$. Ethylene-vinyl acetate copolymer (EVAc, $40 \%$ vinyl acetate, Dupont Co., Wilmington, DE), was washed in distilled water, and then extracted against acetone to remove impurities (36). Heparin (Choay No. 1453, $12,000-18,000 \mathrm{D}, \mathrm{USP} 160 \mathrm{U} / \mathrm{mg}$ ) previously reported to inhibit SMC proliferation in tissue culture (9), and intimal hyperplasia in vivo (11) was obtained from Choay Industries, Paris, France. Lipopolysaccharide (LPS) was from Sigma Chemical Co., St. Louis, MO.

Mouse IgG monoclonal antibody to proliferating nuclear antigen (PCNA) was from Coulter Immunology, Hialeah, FL, and rabbit IgG monoclonal antibody raised against human von Willebrand's factor (factor VIII-related antigen) from Dako Corp., Santa Barbara, CA. Avidin-peroxidase complex $\mathrm{ABC}$ kit, $\mathrm{ABC}$-alkaline phosphatase kit and biotinylated goat and horse anti-rabbit IgG were from Vector Laboratories, Inc., Burlingame, CA. 3,3'-Diaminobenzidine was obtained from Sigma Chemical Co.

Microcapsule encapsulation of $b F G F$. bFGF was ionically bound to heparin-Sepharose beads encapsulated within calcium alginate microcapsules using a modification of a technique previously described (37). This provided a stable platform for the bFGF, enabled prolonged storage, and established a means for the controlled release of bFGF to specific sites in vivo. Heparin-Sepharose beads $(3.33 \mathrm{mg} / \mathrm{ml})$ were sterilized under ultraviolet light for $30 \mathrm{~min}$ and then mixed with filter sterilized sodium alginate $(1.2 \% \mathrm{wt} / \mathrm{vol})$. The mixed slurry was dropped through a needle into a beaker containing a hardening solution of calcium chloride $(1.5 \% \mathrm{wt} / \mathrm{vol})$. Microcapsules were formed instantaneously as the mixture entered the hardening solution. Uniformly cross-linked capsule envelopes were obtained by incubating the capsules in the calcium chloride solution for 5 min under gentle mixing, followed by $10 \mathrm{~min}$ in the solution without mixing. The microcapsules were then washed three times with sterile water and stored in saline containing $1 \mathrm{mM} \mathrm{CaCl}_{2}$ at $4^{\circ} \mathrm{C}$ for an indefinite period of time without degradation (37). Each microcapsule contained $0.5 \mathrm{mg}$ of heparin-Sepharose, 0.36 alginate, and $11 \mathrm{mg}$ of water in its hydrated state. bFGF was incorporated within the microcapsules, after calcium alginate matrix formation and hardening, by incubation in PBS with $0.05 \%$ gelatin, bFGF, and trace amounts of ${ }^{125}$ I-bFGF $(10 \mu$ l per microcapsule) for $16 \mathrm{~h}$ under gentle agitation at $4^{\circ} \mathrm{C}$. Release kinetics were defined by examining microcapsules residing within a large volume $(6 \mathrm{ml}$ per bead) of PBS with $0.05 \%$ gelatin under gentle shaking at $37^{\circ} \mathrm{C}$. The solution was changed on a regular basis and the presence of iodinated tracer was compared to the ability of released bFGF to stimulate thymidine incorporation in BALBc/3T3 cells (38).

Encapsulation of heparin and lipopolysaccharide. Heparin and LPS were solvent cast encapsulated within EVAc matrices as previously described $(11,36)$. Dry powdered drug was added to a solution of EVAc dissolved in dichloromethane $(10 \% \mathrm{wt} / \mathrm{vol})$ to achieve a final ratio of $33 \% \mathrm{wt} / \mathrm{wt}$. The drug-polymer suspension was poured into precooled glass molds, removed after hardening, and placed at $-20^{\circ} \mathrm{C}$ and then under vacuum (600 mtorr) for $2 \mathrm{~d}$ each. The resultant matrix was a homogeneous dispersion of drug within a porous network of EVAc (36). Smaller pellets were cut from the larger slabs and coated with a layer of EVAc. Drug release was restrained to emanate from a hole in the coating and near zero-order kinetics obtained in this fashion $(11,39)$.

Intimal hyperplasia and SMC proliferation. Endothelial denudation of the left common carotid artery in the rats was performed with a 2 French Fogarty balloon catheter (40). Rats were anesthetized with intraperitoneal sodium Nembutal $0.05 \mathrm{mg} / \mathrm{g}$ of body weight. A midline incision exposed the distal left common and external carotid arteries. The balloon catheter was introduced into the external carotid artery and passed three times ("3-pass") with the balloon distended sufficiently with air to generate slight resistance. Inflation pressures were monitored and kept constant with a manometer placed in series circuit with the catheter and air supply. To obtain lesser degrees of vascular injury and resultant proliferation in some animals only a single pass ("1-pass") of the balloon was used. Upon removal of the catheter the external carotid artery was ligated. The contralateral artery underwent identical manipulation save for the introduction of the balloon catheter. Microcapsules and/or matrices were placed adjacent to the arteries and maintained in place by suturing closed fascial planes over the artery (11). Drug was released from these devices directly to the arterial wall. On the 14th postoperative day animals were euthanized and perfused clear retrograde via the abdominal aorta with lactated ringers followed by fixation with modified Ito-Karnovsky's or Carnoy's fixative. The location of the implanted devices was noted and the devices recovered with the intact arteries. Representative sections from both common carotid arteries were processed and stained with Verhoeff's elastin stain, monoclonal antibodies to SMC, PCNA, or von Willebrand's factor. The intimal, medial, and adventitial areas, the intimal/ medial area ratio $(\mathrm{I} / \mathrm{M})$, and the percentage of luminal occlusion were calculated for each arterial segment using computerized digital planimetry. Cell density within each vascular wall compartment was determined by counting the number of cells and dividing by the compartment area. The area and number of blood vessels in the arterial sections were similarly evaluated. In separate experiments vascular permeability was examined by injecting Evan's blue dye $(60 \mathrm{mg} / \mathrm{kg}) 1 \mathrm{~h}$ prior to harvest.

Immunocytochemistry. Sections were stained using antibodies to PCNA to delineate proliferating cells, SMC actin to identify SMCs, and von Willebrand's Factor to distinguish endothelial cells from luminal SMC, and to enhance discrimination of the microvasculature. Sections were deparaffinized in xylene and ethanol baths. Endogenous peroxidase activity was quenched with 20 -min immersion in a solution of 200 $\mathrm{ml}$ of methanol with $50 \mathrm{ml}$ of $3 \%$ hydrogen peroxide. Nonspecific antibody binding was prevented by preincubating the tissues for $20 \mathrm{~min}$ with serum (1:10) from species other than those used to raise the primary antibody. The sections were then exposed to the primary antibody to SMC and von Willebrand's factor for $60 \mathrm{~min}$ and $90 \mathrm{~min}$ for PCNA. Mouse IgG anti-PCNA was used at 1:2,000, mouse anti-SMC actin was used at 1:5,000, and rabbit IgG anti-human von Willebrand's antigen was added at 1:500 and 1:1,000 concentrations. After the sections were rinsed in PBS they were incubated for an additional hour with biotinylated IgG directed against the secondary antibody. Peroxidase labeling was achieved with a 1-h incubation using avidinperoxidase complex. Antibody visualization was established after a 5min exposure to $0.05 \% 3,3^{\prime}$-diaminobenzidine in $0.05 \mathrm{M}$ Tris- $\mathrm{HCl}$ at pH 7.6 with $0.003 \%$ hydrogen peroxide. In other sections antibody visualization was obtained with alkaline phosphatase. After incorpora- 
tion of the secondary antibody was complete, slides were rinsed and soaked in PBS, incubated for $1 \mathrm{~h}$ with ABC-alkaline phosphatase solution, rinsed again with $\mathrm{PBS}$, and exposed to $\mathrm{ABC}$-alkaline phosphatase substrate diluted in $5 \mathrm{ml}$ of Tris- $\mathrm{HCl}$ at $\mathrm{pH} 8.2$ for $10-30 \mathrm{~min}$. Sections were counterstained with methyl green or hematoxylin.

Angiogenesis. Cross sections of the retrieved carotid arteries contained the adjacent alginate microcapsule. Staining with von Willebrand's factor antibody enhanced delineation of blood vessels within these capsules. The number and area of blood vessels were determined using computerized digital planimetry and these were divided by the surface area of the capsule. This provided values for the density of blood vessels in number of vessels per square millimeter, and the average area of neovascular structures within each capsule cross section.

A clear angiogenic response was noted to encircle the artery within a distance equal to the width of the blood vessel wall including the adventitia, media, and neointima. At each point along the circumference of the blood vessel the distance to the lumen was measured and then used to create an external boundary in the opposite direction (see Fig. 1). This circumferential analysis corrected for eccentric intimal hyperplasia, or any asymmetry to the final resting state of the perfused artery. The blood vessels noted within this boundary were considered vasa vasorum. Computer-aided digital planimetry was utilized to sum their number and area. These calculations were performed for both the native (right) carotid and denuded (left) carotid artery in each animal and the difference reported. This differential technique has been used in determining the net increase in cell synthesis after balloon injury (40) and corrected for interanimal variation in baseline number of microvessels. Vessels outside of the bounded area were considered separately, and designated as periimplant angiogenesis and not vasa vasorum (Fig. 1).

Statistics. Statistical comparisons were performed with non-paired t-test for groups of unequal sample sizes, or analysis of variance (ANOVA) when appropriate. Data was rejected as not significantly differ- ent if $P$ values of $>0.05$ were observed. Data line fits were established using a linear regression and correlation model.

\section{Results}

Release kinetics. bFGF incorporation within the alginate microcapsule proceeded with $80 \%$ efficiency and each capsule contained $\sim 800 \mathrm{ng}$ of bFGF. Prolonged first-order release kinetics were obtained (Fig. 2), with retention of $90.2 \pm 3.6 \%$ of the biological activity of the released bFGF. It is estimated that during the linear portion of the release curve the implanted capsules release $\sim 34 \mathrm{ng} / \mathrm{d}$ to the arterial wall, such that over the course of the 14-d experiment at most $500 \mathrm{ng}$ of bFGF would have been delivered to the blood vessel wall.

Effect of bFGF on intimal hyperplasia and SMC proliferation. bFGF is a SMC mitogen in tissue culture. It is possible, therefore, that the perivascular controlled release of bFGF might enhance the SMC response after mechanical de-endothelialization, or induce SMC proliferation in an artery with intact endothelium. When bFGF was released from alginate microcapsules adjacent to ("3-pass") denuded carotid arteries, there was a slight increase in intimal hyperplasia. In the 17 bFGF-treated animals an I/M of $1.28 \pm 0.09$ was obtained in the injured artery (Table I and Figs. 3 and 4). In contrast, the 13 animals with blank microcapsules exhibited an I/M of $0.96 \pm 0.08$ (blank vs. bFGF, $P<0.018$, ANOVA). There was, however, no hyperplasia in the absence of balloon injury to the arterial wall; bFGF did not induce any SMC proliferation in native arteries or arteries subjected to mock surgery with all steps aside from balloon inflation and withdrawal. When me-

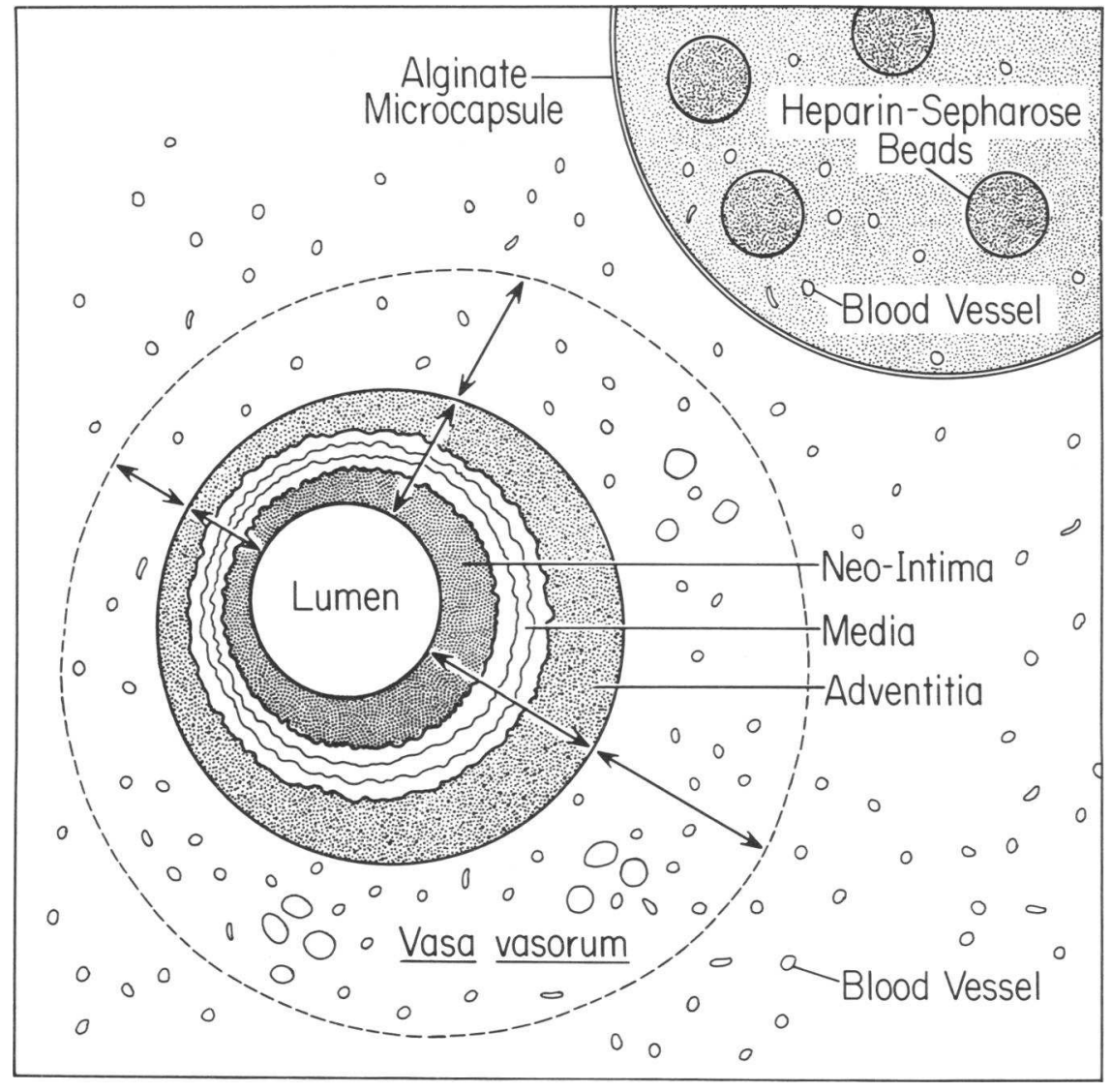

Figure 1. Schematic diagram of carotid artery with an adjacent alginate microcapsule implant demonstrates the three zones of microvascular proliferation in response to the controlled release of bFGF. New blood vessels were noted in the alginate microcapsule, in the area between the artery and the microcapsule, and surrounding the artery. The vessels within this last region were distinctly different from the others and increased in number in a manner directly related to the extent of neointimal proliferation. All blood vessels within this zone were defined as vasa vasorum. The boundary of the region containing vasa was defined at any given radial direction as equal to twice the distance from the arterial lumen to the outer adventitia, as measured from the center of the artery. 


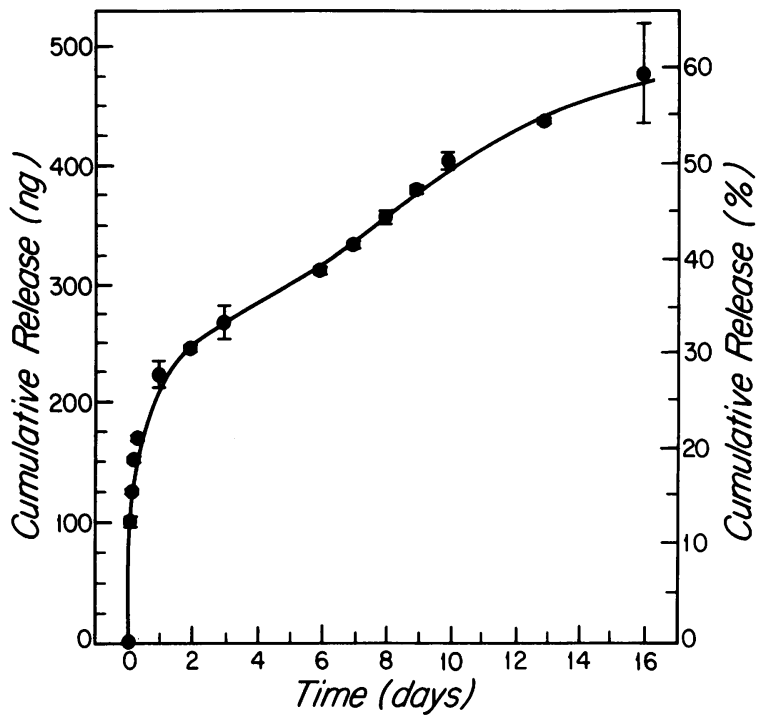

Figure 2. bFGF release kinetics from alginate microcapsules reveal prolonged zero order release after an initial burst of release in the first $24 \mathrm{~h}$. Cumulative bFGF release is plotted as an absolute amount in nanograms, and as a percentage of the total amount of growth factor incorporated within the alginate microcapsule. Each point represents the mean \pm SEM for three identical microcapsules. Over the 14-d period of the experiment $\sim 500 \mathrm{ng}$ of bFGF was released from the capsules. The growth factor was released biologically intact with retention of $90.2 \pm 3.6 \%$ of the expected biological activity.

chanical injury was performed with the intent of inducing lesser degrees of vascular injury ("1-pass") an I/M of $0.13 \pm 0.03$ was noted ( $n=11$, Table I and Fig. 4). When bFGF was applied to 10 animals injured in this fashion, intimal proliferation was increased 2.4-fold to $0.31 \pm 0.04$ (blank vs. bFGF, $P<0.002$, ANOVA).

To examine whether the bFGF mediated increase in intimal mass was the result of additional production of matrix material or an increase in the number of proliferating cells we determined the density of cells within the intima, and the number of proliferating cells at the time of harvest. Though there was a significant difference in intimal proliferation between the control and bFGF-treated injured blood vessels intimal cell density was unchanged (Table I), implying that there was no significant increase in extracellular mass. The number of intimal cells possessing PCNA antigenicity increased equivalently to the increase in intimal area, irrespective of the degree of injury (Table I). The absolute number of cells staining for PCNA was greatest in those sections obtained from animals subjected to standard (3-pass) ballooned injury. Yet, the relative increase in the number of proliferating cells was greater in those animals subjected to a single passage of the balloon when compared to the triple passage-treated animals (1.9- vs. 4.0fold increase above baseline, $P<0.038$ ). There was no statistical difference in the number of proliferating cells within the media at tissue harvest 2 wk after vascular injury.

Concomitant effect of heparin and $b F G F$. We have previously demonstrated that perivascular heparin suppresses intimal hyperplasia and SMC proliferation after balloon injury by 83.2\% (11). bFGF has a well-defined affinity for heparin and has been characterized and purified by passage over columns with immobilized heparin $(30,34,35)$. Heparin protects bFGF from degradation and denaturation (41). To determine whether bFGF might interfere with the ability of heparin to inhibit hyperplasia or whether heparin would potentiate the bFGF effects, the two drugs were administered simultaneously to the perivascular space of five animals. In five different animals bFGF microcapsules were accompanied by blank EVAc matrices. All animals were subjected to endothelial denudation
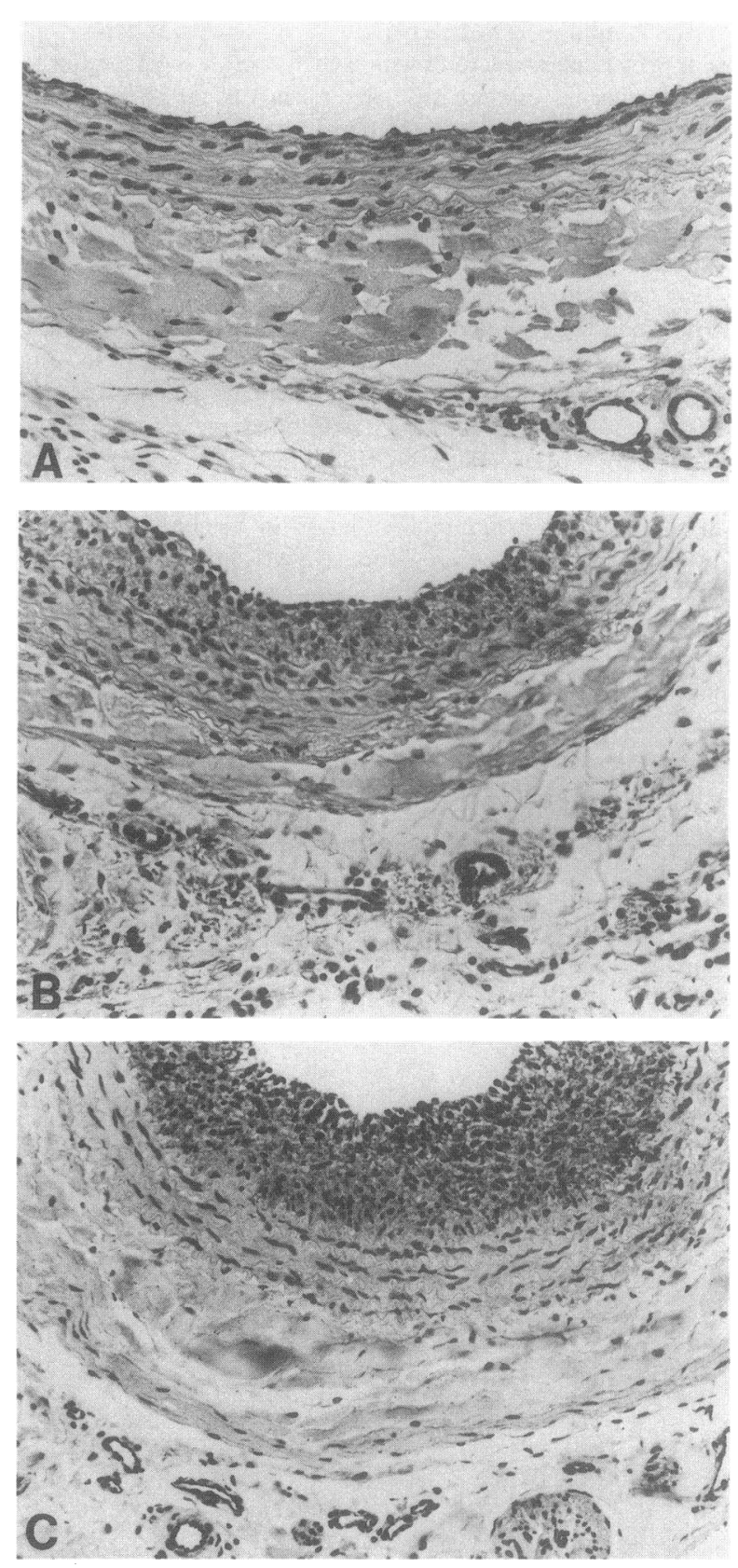

Figure 3. Photomicrographs $(\times 250)$ of perivascular tissue demonstrate the vascular response to bFGF. $(A)$ There is no intimal hyperplastic response, and there is little demonstrable perivascular angiogenesis in surrounding arteries exposed to bFGF and subjected only to sham operation without balloon inflation. $(B)$ In arteries subjected to balloon injury neointimal hyperplasia accompanied by SMC proliferation is evident, but little vascular proliferation is noted. $(C)$ In contrast, when arteries are balloon injured and treated with bFGF both the SMC and microvascular proliferative responses are enhanced. 


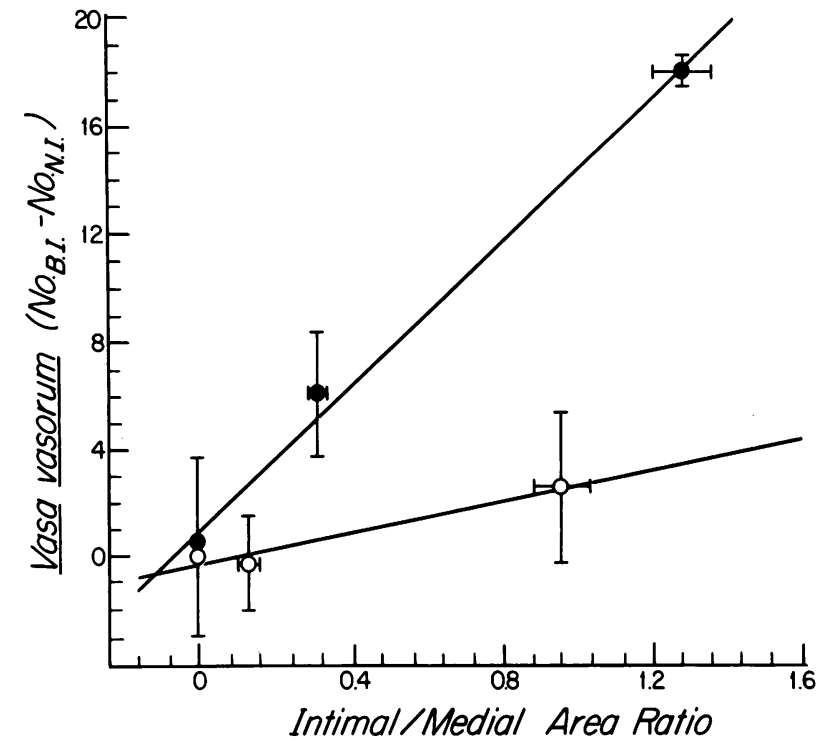

Figure 4. Stimulation of vasa vasorum is linked to the degree of intimal hyperplasia in bFGF-treated injured blood vessels. The average difference between the number of vasa vasorum in the ballooned (B.I.) and sham-operated, noninjured (N.I.) arteries is plotted, for bFGF-treated ( $\bullet$ ) and untreated animals (O), against the intimal/medial area ratio in the injured artery. The line fits join the points representing no injury, single-pass balloon injury and triple-pass balloon injury. Data is represented as the mean \pm SEM. Error bars are presented in both the abscissal and ordinal directions.

with three passes of the inflated balloon catheter. Significant intimal proliferation was noted in those animals receiving bFGF alone; an I/M of $1.42 \pm 0.12$ was obtained. Heparin reduced this proliferation $83.8 \%$, and the $\mathrm{I} / \mathrm{M}$ was $0.23 \pm 0.13$.

LPS-induced vascular injury. The endotoxin, LPS, has been administered intravenously and directly to the vascular wall to induce endothelial injury $(42,43)$. We chose to examine whether endothelial injury by LPS in the absence of mechanical injury might permit bFGF-stimulated SMC proliferation. EVAc matrices containing LPS were placed adjacent to the left carotid artery of five rats, and blank EVAc matrices placed on the right. All LPS-treated arteries demonstrated a marked increase in permeability to Evan's blue, and rich vascularization in the area of the EVAc matrix, but no detectable intimal proliferation. bFGF had no additional affect on these arteries in the presence of LPS. Alginate microcapsules containing bFGF were placed adjacent to both of the carotid arteries in a separate set of five animals treated as above. Thus, the left carotid artery was subjected to the simultaneous sustained release of LPS and bFGF from two different devices, while the right artery was surrounded by both a blank EVAc matrix and a microcapsule releasing bFGF. None of the arteries displayed any neointimal proliferation, alteration in staining for SMC actin, or difference in the angiogenic response to LPS. The number of microvasculature per square millimeter surrounding arteries treated with the blank EVAc matrix and bFGF was 11.4 \pm 1.6 , and around those receiving LPS and bFGF, $11.8 \pm 2.7(P=\mathrm{NS}$, ANOVA).

Angiogenic effect of released $b F G F$. The angiogenic potential of bFGF is well documented. Implanted sponges, gels or beads soaked in bFGF were richly and rapidly vascularized (19, 22-25). We counted the number of blood vessels within the alginate microcapsules (Fig. 1), containing heparin-Sepharose
Table I. Intimal Hyperplasia and Cell Proliferation

\begin{tabular}{lccc}
\hline & $\mathrm{I} / \mathrm{M}$ & Cell density & PCNA+ \\
\hline 1-pass balloon injury & & & \\
Control $(n=11)$ & $0.13 \pm 0.03$ & $10,362.4 \pm 665.2$ & $1.2 \pm 0.4$ \\
bFGF $(n=10)$ & $0.31 \pm 0.03^{*}$ & $10,942.2 \pm 623.8$ & $4.8 \pm 1.6^{\ddagger}$ \\
bFGF:Control & 2.4 & 1.1 & 4.0 \\
& $* P<0.002$ & & ${ }^{\ddagger} P<0.035$ \\
3-pass balloon injury & & & \\
Control $(n=13)$ & $0.96 \pm 0.08$ & $9,422.5 \pm 445.6$ & $19.7 \pm 4.1$ \\
bFGF $(n=17)$ & $1.28 \pm 0.09^{\S}$ & $9,533.3 \pm 419.9$ & $37.1 \pm 5.1^{11}$ \\
bFGF:Control & 1.3 & 1.0 & 1.9 \\
& $\$ P<0.02$ & & $" P<0.038$ \\
& & & \\
\hline
\end{tabular}

Intimal medial area ratio $(\mathrm{I} / \mathrm{M})$, cell density expressed as the total number of cells per intimal area $\left(\mathrm{mm}^{2}\right)$, and the number of proliferating cells (PCNA+) per neointimal cross section at tissue harvest, 2 wk after vascular injury, under control conditions and with bFGF therapy, for triple- and single-pass balloon injury.

alone or heparin-Sepharose and bFGF, and measured the areas of these blood vessels as well. While there were virtually no blood vessels within the control microcapsules without bFGF (Table II), those microcapsules that contained and released the growth factor were heavily vascularized (Table II). Alginate capsules with bFGF contained 6.9 times the number of blood vessels as their blank counterparts. Moreover, the area of the blood vessels within the bFGF laden capsules was 2.9 times greater than the area of corresponding blood vessels in the blank capsules. This specific angiogenic effect of bFGF was independent of the degree of injury, as there was no statistical difference between the number or area of vessels in microcapsules laid adjacent to noninjured, "1-pass"-injured, or "3pass"-injured blood vessels. Thus, bFGF induced growth of more abundant and larger blood vessels within the alginate

Table II. Neovascularization of the Alginate Microcapsules

\begin{tabular}{lcc}
\hline & Vascular density & Average vessel area \\
\hline & number vessels $/ \mathrm{mm}^{2}$ & $\mu \mathrm{m}^{2}$ \\
Control & $4.3 \pm 0.1$ & $66.1 \pm 1.7$ \\
bFGF total & $29.5 \pm 2.8^{*}$ & $189.0 \pm 40.3^{\ddagger}$ \\
bFGF:Control & 6.9 & 2.9 \\
& $* P<0.001$ & ${ }^{*} P<0.005$ \\
bFGF-treated & & \\
Noninjured & $32.8 \pm 4.2$ & $218.3 \pm 84.1$ \\
Balloon injury & & \\
$\quad$ 1-pass & $26.7 \pm 8.7$ & $209.9 \pm 42.9$ \\
3-pass & $30.2 \pm 5.2$ & $174.1 \pm 45.1$ \\
\hline
\end{tabular}

Density and average area, of blood vessels within alginate microcapsules embedded with heparin-Sepharose beads alone (control) or with bFGF-laden heparin-Sepharose beads (bFGF). The realtive increase in each parameter is displayed as the ratio of bFGF to control values. Data for the bFGF-treated animals is further subdivided to include analysis of capsules placed adjacent to noninjured blood vessels, and vessels subjected to 1- or 3-pass denudation with an inflated balloon catheter. 
microcapsular growth factor-releasing devices irrespective of the state of the adjacent artery.

In contrast in the area immediately surrounding the carotid artery bFGF neovascularization was dependent on the amount of neointimal hyperplasia. Blood vessels were counted within a width of the carotid artery's wall, including the media and neointima, and termed vasa vasorum (Fig. 1). The number of vasa discovered surrounding the left, balloon-injured, carotid artery was subtracted from the number of vasa surrounding the uninjured, right carotid artery. At every level of injury there were more vasa in the bFGF-treated group. With increasing injury the difference in vasa counted in the treated and untreated groups rose. In the series of animals treated with bFGF there was linear relationship between the number of vasa and the extent of intimal hyperplasia (slope $=13.5, R^{2}=0.993, P$ $=0.008$ ). In the control treated animals a linear relationship was also observed, but there was no statistical significance between the number of vasa at different points (slope $=3.0, R^{2}$ $=0.959, P=\mathrm{NS}$ ).

Endothelial regeneration. The distribution of Evan's blue after intravenous injection one hour prior to harvest illuminated gross alterations in vascular permeability and/or endothelial regeneration. The dye passed into the wall of all ballooned carotids regardless of therapy. There was no difference in the length of the blued segments between the control and bFGFtreated groups. None of the sham operation carotids allowed dye to permeate into the wall. Microscopic evaluation of endothelial state was assessed by staining arterial cross sections with antibodies to von Willebrand's factor. Intact endothelium was observed in all of the nondenuded arteries and in all blood vessels surrounding the arteries. None of the sections obtained from ballooned vessels demonstrated endothelialization of the injured segments, whether treated with bFGF or control capsules.

\section{Discussion}

bFGF is synthesized by and mitogenic for a variety of cell lines including endothelial cells and SMCs (19-21), and is intensely angiogenic $(19,22-25)$. Intravenous infusion of bFGF stimulated endothelial regeneration (33) and SMC proliferation (31) after balloon-induced endothelial denudation. The effect on SMC was noted only in the presence of endothelial denudation. It was concluded that the endothelium may serve as a barrier preventing contact of the mitogen, bFGF, with the target, intimal SMC (31). We confirmed that bFGF was both angiogenic and mitogenic for SMC in vivo, and demonstrated that these two effects were coupled. These phenomena, and the absence of intimal hyperplasia in the nondenuded blood vessel exposed to bFGF from the periadventitial aspect of the blood vessel, may indicate that the endothelium may be serving as more than a physical barrier and lends further support to the hypothesis that the endothelium is chemically inhibiting SMC proliferation in the quiescent state.

Neointimal hyperplasia, SMC proliferation, and bFGF. bFGF is a complex growth factor with a myriad of potential vasoactive activities. Its ability to stimulate growth of cells and vascular tissues may involve both direct and indirect mechanisms. Platelet aggregation (44) and vascular tone (45) are both increased by this growth factor. We have confirmed that
bFGF's growth stimulatory activity on SMC in vivo seemingly requires loss of endothelium. When arterial injury was performed with three passes of the inflated balloon catheter, bFGF induced a 1.3-fold increase in the intimal mass. If lesser baseline injury was obtained the net increase was 2.4-fold. PCNA positivity and cell density indicate that the net increase in intimal mass was the result of cell proliferation, and not simply an increase in extracellular matrix. The density of cells per unit intimal area remained constant indicating no increase in noncellular elements, and the number of proliferating cells increased on the same order of magnitude as the increase in intimal mass. As with the increase in intimal mass the relative increase in the number of proliferating cells within the intima of the lesser injured arteries was approximately twofold greater than with injury that induced greater baseline hyperplasia.

These data suggest that the in vivo mitogenic potential of bFGF for SMC is complex: present only with preexistent endothelial denudation but relatively more effective at lower levels of endothelial and SMC injury. These observations might explain why bFGF, while ubiquitous, does not cause significant angiogenesis or SMC proliferation in the quiescent state. Some have proposed that the endothelium may serve as a mechanical barrier for bFGF (31). Others have hypothesized that local injury is required for the release of bFGF or the removal of bFGF inhibitors $(26-28,34)$. We believe that our study lends further support to the latter hypothesis. Progressive balloon injury may remove increasingly more bFGF inhibitor, such as endothelial associated heparan sulfate, or free more endogenous bFGF, permitting SMC proliferation and inducing an angiogenic signal. These observations and hypotheses do not infer that all SMC proliferation is blocked in the presence of endothelium, and do not preclude SMC proliferation even in our model beyond the limit of the sensitivity of our immunocytochemical assay or at some period of time other than during which we examined the arterial sections. Furthermore, one cannot even say with certainty that endothelial loss alone is the causal factor for SMC replication after balloon injury. It is possible that a multitude of vascular effects are induced by the balloon catheter, only one of which is endothelial denudation. Because the endothelium produces a factor known to inhibit smooth muscle cell proliferation, it is easy to speculate that alterations in the metabolism of this factor are responsible for alterations in the intimal hyperplastic response to vascular injury. Endothelial denudation, however, may only be an epiphenomenon accompanying some other event. Increased SMC proliferation itself might be accompanied by the increased production of as yet undefined factors that potentiate the binding or activity of bFGF. The exposure of basement membrane with increasing amounts of vascular injury might also play a role by exposing bFGF to circulating cells and/or secreted factors (26-29, 34, 46, 47).

"Minimal" vascular injury has been used to examine a number of elements of the blood vessel biology (48-50). When vascular endothelial cell removal was accomplished without apparent effect on the media no SMC proliferation was noted in the blood vessel wall. This suggested that neointimal proliferation is determined not so much by endothelial injury but rather by the extent of damage to the SMC containing media (48-50). Dilating injury of the rat carotid artery increased intimal hyperplasia five times more than denuding injury (51), and the degree of dilation was an important determinant of the 
extent of proliferation $(51,52)$. In our experiments single passage of the inflated balloon denuded the artery of endothelium and reproducibly produced only $13.5 \%$ of the increase in intimal mass as with three passes of the balloon catheter. Yet, both I/M and PCNA positivity showed a greater bFGF responsiveness in comparison to corresponding controls in the lesser injured blood vessels. The I/M after single-pass injury was 1.9fold higher than that observed after triple injury, and the PCNA positivity was 2.1 -fold increased. It is possible that the more severe form of balloon injury induces a maximal or nearmaximal SMC response and only marginal additional stimulation can be achieved after that. When lesser degrees of injury were induced, as with a single pass of the balloon, the vascular SMC might then have been more amenable to growth promotion.

In the other extreme, periadventitial release of LPS induced a "minimal" injury with marked increase in vascular permeability without loss of endothelium. Administration of bFGF to arteries treated in this fashion induced no intimal proliferation, increased neovascularization or alteration in the medial SMC. The administration of LPS alone also had no effect. Variable effects on vascular healing have been reported with LPS in the past. Reidy et al. (42) noted that intravenous injections of LPS enhanced endothelial cell replication without change in endothelial cell density or SMC proliferation (42). In contrast, Prescott et al. (43) wrapped a LPS soaked suture and a "cleaned" artery within a polyethylene film, and observed intimal SMC proliferation, and micro-vascular propagation within the adventitia. It is possible that "cleaning" or wrapping the artery releases a signal necessary for LPS induction of SMC proliferation, and that bFGF relies on the SMC themselves or the signal that drives their proliferation to generate specific bFGF mitogenic and angiogenic effects.

Heparin and $b F G F$. Heparin has a remarkable range of physiologic effects. The intravenous $(3,10)$ and periadventitial (11) administration of heparin suppresses SMC proliferation after balloon arterial injury. In addition, bFGF has a well-defined affinity for heparin and has been characterized and purified by passage over columns with immobilized heparin (30, $35,53)$. Heparin prevents losses in bFGF activity that accompany changes in $\mathrm{pH}$, elevation in temperature, or chemical processes that lead to denaturation (41). Thus, one might observe either mutual inhibition or potentiation with the concomitant use of these compounds. In fact, heparin suppressed intimal hyperplasia and SMC proliferation when co-administered with bFGF to an identical extent as when bFGF was not present (11). This may be related to the relative doses of the two drugs. We utilized milligram quantities of heparin, as this is the dose range that inhibits SMC proliferation after injury, and nanogram to microgram quantities of bFGF. Equimolar concentrations of heparin and bFGF may have far different effects. Similarly, Sepharose-immobilized heparin may stabilize bFGF sufficiently with little benefit from additional heparin. It should be noted, however, that little to none of the immobilized heparin is released from the alginate capsules (37), and that alginate capsules without bFGF did not alter intimal hyperplasia or SMC proliferation.

Perivascular angiogenesis. The angiogenic potential of bFGF is well documented. In our experiments it appears that this phenomena may have two distinct components. First, angiogenesis in areas of highest bFGF concentration, within the controlled release device and immediately surrounding it, was not related to intimal hyperplasia within the adjacent blood vessel. This is the type of angiogenesis described previously (19, 22-25). In the presence of moderate concentrations of bFGF and proximity to an injured artery a second type of vascular response was noted. The microvasculature generated was concentrated within a zone equal to the width of the blood vessel wall, including the neointima. However, unlike the angiogenesis within the capsule and surrounding tissue, the number of perivascular vessels increased as the degree of injury increased in the local carotid artery. These vessels fit the classical definition of vasa vasorum, which grow in reaction to an increase in arterial wall thickness beyond a width that can be nourished by luminal perfusion. As such it is not entirely surprising that they are controlled by the state of the larger vessel. Though the linkage between angiogenesis and mitogenesis seems clear, it is not evident whether one drives the other. It is intriguing, for example, that blocking angiogenesis might block intimal hyperplasia, and vice versa. Yet, it may be difficult to isolate one effect from the other as many agents that exhibit an effect on one of these axes have also been reported to possess activity on the other axis.

Periadventitial bFGF release. The in vivo half-life of bFGF has been estimated to be in the range of 2-4 min (54), and this growth factor is remarkably prone to denaturation and degradation $(41,47)$. As a result a variety of investigators have used some form of controlled administration to sustain release and limit the amount of bFGF used $(24,25,55)$. Alginate microcapsules were used in our experiments because release kinetics could be tailored (37) and prolonged (Fig. 2). Other systems tend to release the bulk of the bFGF within the first $96 \mathrm{~h}$. The alginate microcapsules delivered 1/300th the amount of bFGF infused in intravenous studies (33) which may account for differences in data. Direct application to the periadventitial space may also have influenced our results. The adventitia is rich in perivascular structures which have been suggested to provide access to or control of the media and intima (12-15). In addition, the rich vascularization surrounding the intimal hyperplastic lesion may permit more efficient exposure of the cells to the growth factor. We have previously defined the release kinetics for this type of administration. Normal and denuded arteries were exposed in a circumferentially symmetric fashion to compounds, of similar molecular weight to bFGF, released in this manner (11). Hence, it may well be that bFGF is most effective when administered in the perivascular region of the injured blood vessel rather than in a manner that would require transit through the entire vascular system first.

Thus, it seems that mitogenic and angiogenic effects of exogenous bFGF on the injured artery are linked. These individual responses and the extent of their correlation are related to the amount of injury to the involved blood vessel. The bFGF stimulation of neovasculature and neointima was greatest when lesser baseline injury was applied to the blood vessel, but only if the injury was significant enough to denude endothelium. If the endothelium was left intact or simply made highly permeable there was no effect of the growth factor. The coupling of the angiogenic and mitogenic responses to vascular injury suggests a synergistic effect between the growth factors that are produced, secreted, or bound to the blood vessel wall, and the SMC that proliferate there. Perhaps modulation of one response might be used to control the other. 


\section{Acknowledgments}

We thank Robert Rubin for photographic assistance.

This work was supported by grants from the National Institutes of Health (HL 17747) and the Massachusetts Affiliate of the American Heart Association (grant 13-524-889). Dr. Edelman is a recipient of a Physician-Scientist Award of the National Institutes of Health (K12 AG00294), and Dr. Nugent is recipient of a National Institutes of Health fellowship (F32 GM14003).

\section{References}

1. Liu, M. W., G. S. Roubin, and B. K. Spencer III. 1989. Restenosis after coronary angioplasty: potential biologic determinants and role of intimal hyperplasia. Circulation. 79:1374-1387.

2. Ip, J. H., V. Fuster, L. Badimon, J. Badimon, M. B. Taubman, and J. H. Chesebro. 1990. Syndromes of accelerated atherosclerosis: role of vascular injury and smooth muscle proliferation. J. Am. Coll. Cardiol. 15:1667-1687.

3. Clowes, A. W., and M. J. Karnovsky. 1977. Suppression by heparin of smooth muscle cell proliferation in injured arteries. Nature (Lond.). 265:625626.

4. Jonasson, L., J. Holm, and G. K. Hansson. 1988. Cyclosporin A inhibits smooth muscle cell proliferation in the vascular response to injury. Proc. Natl. Acad. Sci. USA. 85:2303-2306.

5. Handley, D. A., R. G. Van Valen, M. K. Melden, and R. N. Saunders. 1986. Suppression of rat carotid lesion development by the calcium channel blocker PN-200-110. Am. J. Pathol. 124:88-93.

6. El-Sanadiki, M. N., K. S. Cross, J. J. Murray, R. W. Schuman, E. Mikat, R. L. McCann, and P.-O. Hagen. 1990. Reduction of intimal hyperplasia and enhanced reactivity of experimental vein bypass grafts with verapamil treatment. Ann. Surg. 212:87-96.

7. Powell, J. S., J.-P. Clozel, R. K. M. Muller, H. Kuhn, E. Hefti, M. Hosang and H. R. Baumgartner. 1989. Inhibitors of angiotensin-converting enzyme prevent myointimal proliferation after vascular injury. Science (Wash. DC). 245:186-189.

8. Liu, M. W., G. S. Roubin, K. A. Robinson, A. J. R. Black, J. A. Hearn, R. J. Siegel, and B. K. Spencer III. 1990. Trapadil in preventing restenosis after balloon angioplasty in the atherosclerotic rabbit. Circulation. 81:1089-1093.

9. Castellot, J. J., Jr., T. C. Wright, and M. J. Karnovsky. 1987. Regulation of vascular smooth muscle cell growth by heparin and heparan sulfate. Semin. Thromb. Hemostasis. 13:489-503.

10. Guyton, J. R., R. D. Rosenberg, A. W. Clowes, and M. J. Karnovsky. 1980. Inhibition of rat arterial smooth muscle cell proliferation by heparin I. In vivo studies with anticoagulant and non-anticoagulant heparin. Circ. Res. 46:625-634

11. Edelman, E. R., D. A. Adams, and M. J. Karnovsky. 1990. Effect of controlled adventitial heparin delivery on smooth muscle cell proliferation following endothelial injury. Proc. Natl. Acad. Sci. USA. 87:3773-3777.

12. Geiringer, E. 1951. Intimal vascularization and atherosclerosis. J. Pathol. Bacteriol. 63:201-211.

13. Barger, A. C., R. Beeuwkes III, L. L. Lainey, and K. J. Silverman. 1984 Hypothesis: Vasa vasorum and neovascularization of human coronary arteries. N. Engl. J. Med. 310:175-177.

14. Diaz-Flores, L., and C. Dominquez. 1985. Relation between arterial intimal thickening and the vasa vasorum. Virchow Arch. Pathol Anat. 406:165-177.

15. Zamir, M., and M. D. Silver. 1985. Vasculature in the walls of human coronary arteries. Arch. Pathol. Lab. Med. 109:659-662.

16. Schweigerer, L., G. Nuefeld, J. Friedman, J. A. Abraham, J. C. Fiddes, and D. Gospodarowicz. 1987. Capillary endothelial cells express basic fibroblas growth factor, a mitogen that stimulates their own growth. Nature (Lond.). 325:257-259.

17. Vlodavsky, I., R. Friedman, R. Sullivan, J. Sasse, and M. Klagsbrun. 1987. Aortic endothelial cells synthesize basic fibroblast growth factor which remains cell-associated and platelet-derived growth factor-like protein which is secreted. J. Cell. Physiol. 131:402-428.

18. Winkles, J. A., R. Friesel, W. H. Burgess, R. Howk, T. Mehlman, R. W. R., and T. Maciag. 1987. Human vascular smooth muscle cells both express, and respond to heparin-binding growth factor I (endothelial cell growth factor). Proc. Natl. Acad. Sci. USA. 84:7124-7128.

19. Thomas, K. A., and G. Gimenez-Gallego. 1986. Fibroblast growth factors: broad spectrum mitogens with potent angiogenic activity. Trends Biochem. Sci. 11:81.

20. Presta, M., D. Moscatelli, J. J. Silverstein, and D. B. Rifkin. 1986. Purification from a human hepatoma cell line of a basic FGF like molecule that stimulates capillary endothelial cell plasminogen activator production DNA synthesis and migration. Mol. Cell. Biol. 6:4060.
21. Connolly, D. T., B. L. Stoddard, N. K. Harakas, and J. Feder. 1987. Human fibroblast-derived growth factor is a mitogen and chemoattractant for endothelial cells. Biochem. Biophys. Res. Commun. 144:705.

22. Folkman, J., and M. Klagsbrun. 1985. Angiogenic factors. Science (Wash. DC). 235:442.

23. Montesano, R., J. D. Vassali, A. Baird, R. Guillemin, and L. Orci. 1986. Basic fibroblast growth factor induces angiogenesis in vitro. Proc. Natl. Acad. Sci. USA. 83:7287-7301.

24. Hayek, A., F. L. Culler, G. M. Beattie, A. D. Lopez, P. Cuevas, and A. Baird. 1987. An in vivo model for study of the angiogenic effects of basic fibroblast growth factor. Biochem. Biophys. Res. Commun. 147:876-880.

25. Thompson, J. A., K. D. Anderson, J. M. DiPietro, J. A. Zwiebel, M. Zametta, W. F. Anderson, and T. Maciag. 1988. Site-directed neovessel formation in vivo. Science (Wash. DC). 1349-1352.

26. Baird, A., and N. Ling. 1987. Fibroblast growth factors are present in the extracellular matrix produced by endothelial cells in vitro: implications for a role of heparinase-like enzymes in the neovascular response. Biochem. Biophys. Res. Commun. 142:428-435.

27. Vlodavsky, I., J. Folkman, R. Sullivan, R. Fridman, R. Ishai-Michaeli, J. Sasse, and M. Klagsbrun. 1987. Endothelial cell-derived basic fibroblast growth factor; synthesis and deposition into subendothelial extracellular matrix. Proc. Natl. Acad. Sci. USA. 84:2292-2296.

28. Folkman, J., M. Klagsbrun, J. Sasse, M. Wadzinski, D. Ingber, and I. Vlodavsky. 1988. Heparin-binding angiogenic protein-basic fibroblast growth factor-is stored within basement membrane. Am. J. Pathol. 130:393-400.

29. Presta, M., J. A. M. Maier, M. Rusnati, and G. Ragnotti. 1989. Basic fibroblast growth factor is released from endothelial extracellular matrix in a biologically active form. J. Cell. Physiol. 140:68-74.

30. Klagsbrun, M., and E. R. Edelman. 1989. Biological and biochemical properties of fibroblast growth factors: implications for the pathogenesis of atherosclerosis. Arteriosclerosis. 9:269-278.

31. Lindner, V., D. A. Lappi, A. Baird, R. A. Majack, and M. A. Reidy, 1991. Role of basic fibroblast growth factor in vascular lesion formation. Circ. Res. 68:106-113.

32. Lindner, V., and M. A. Reidy. 1991. Proliferation of smooth muscle cells after vascular injury is inhibited by an antibody against basic fibroblast growth factor. Proc. Natl. Acad. Sci. USA. 88:3739-3743.

33. Lindner, V., R. A. Majack, and M. A. Reidy. 1990. Basic fibroblast growth factor stimulates endothelial regrowth and proliferation in denuded arteries. $J$. Clin. Invest. 85:2004-2008.

34. Bashkin, P., S. Doctrow, M. Klagsbrun, C. M. Svahn, J. Folkman, and I. Vlodavsky. 1989. Basic fibroblast growth factor binds to subendothelial extracellular matrix and is released by heparanase and heparin-like molecules. Biochemistry. 28:1737-1743.

35. Rifkin, D. B., and D. Moscatelli. 1989. Recent developments in the cell biology of basic fibroblast growth factor. J. Cell Biol. 109:1-6.

36. Langer, R., L. Brown, K. Leong, Y. Kost, and E. Edelman. 1985. Controlled release and magnetically modulated systems for macromolecular drugs. Ann. N.Y. Acad. Sci. 446:1-13

37. Edelman, E. R., E. Mathiowitz, R. Langer, and M. Klagsbrun. 1991. Controlled and modulated release of basic fibroblast growth factor. Biomaterials. 12:619-626.

38. Sullivan, R., and M. Klagsbrun. 1987. Purification and assay of intact human basic fibroblast growth factor using heparin-Sepharose chromatography. J. Tissue Cult. Methods. 10:125-132.

39. Rhine, W. D., V. Sukhatme, D. S. T. Hsieh, and R. Langer. 1980. A new approach to achieve zero-order release kinetics from diffusion-controlled polymer matrix systems. In Controlled Release of Bioactive Materials. R. W. Baker, editor. Academic Press, Inc., New York. 177-187.

40. Clowes, A. W., M. A. Reidy, and M. M. Clowes. 1983. Kinetics of cellular proliferation after arterial injury. I. Smooth muscle growth in the absence of endothelium. Lab. Invest. 49:327-333.

41. Gospodarowicz, D., and J. Cheng. 1986. Heparin protects basic and acidic FGF from inactivation. J. Cell. Phys. 128:475-484.

42. Reidy, M. A., and S. M. Schwartz. 1983. Endothelial injury and regeneration. IV. Endotoxin: a nondenuding injury to aortic endothelium. Lab. Invest. 48:25-34.

43. Prescott, M. F., C. K. McBride, and M. Court. 1989. Development of intimal lesions after leukocyte migration into the vascular wall. Am. J. Pathol. 135:835-846.

44. Greisler, H. P., J. J. Klosak, S. J. Steinam, T. M. Lam, W. H. Burgess, and D. U. Kim. 1990. Effects of class I heparin binding growth factor and fibronectin on platelet adhesion and aggregation. J. Vasc. Surg. 11:665-674.

45. Berk, B. C., R. W. Alexander, T. A. Brock, M. A. Jr. Gimbrone, and R. C. Webb. 1986. Vasoconstriction: a new activity for platelet-derived growth factor. Science (Wash. DC). 232:87-90.

46. Moscatelli, D. 1987. High and low affinity binding sites for basic fibroblast growth factor on cultured cells: absence of a role for low affinity binding in the stimulation of plasminogen activator production by bovine capillary endothelial cells. J. Cell. Physiol. 131:123-130. 
47. Gospodarowicz, D., G. Neufeld, and L. Schweigerer. 1987. Fibroblast growth factor: structural and biological properties. J. Cell. Physiol. Supp. 5:1526.

48. Bjorkerud, S. 1969. Reaction of aortic wall of the rabbit after superficial longitudinal mechanical trauma. Virchows Arch. A. Pathol. Anat. Histol. 347:197.

49. Reidy, M. A., and S. M. Schwartz. 1981. Endothelial regeneration. III. Time course of intimal changes after small defined injury to rat aortic endothelium. Lab. Invest. 44:301-308.

50. Reidy, M. A., and M. Silver. 1985. Endothelial regeneration. VII. Lack of intimal proliferation after defined injury to rat aorta. Am. J. Pathol. 118:173-177.

51. Webster, M. W. I., J. H. Chesebro, M. Heras, J. S. Mruk, D. E. Grill, and
V. Fuster. 1990. Effect of balloon inflation on smooth muscle cell proliferation in the porcine carotid artery. J. Am. Coll. Cardiol. 15:165A. (Abstr.)

52. Sarembock, I. J., P. J. LaVeau, S. L. Sigal, I. Timms, J. Sussman, C. Haudenschild, and M. D. Ezekowitz. 1989. Influence of inflation pressure and balloon size on the development of intimal hyperplasia after balloon angioplasty: A study in the atherosclerotic rabbit. Circulation. 80:1029-1040.

53. Burgess, W. H., and T. Maciag. 1989. The heparin-binding (fibroblast) growth factor family of proteins. Annu. Rev. Biochem. 58:575-606.

54. Whalen, G. F., Y. Shing, and J. Folkman. 1989. The fate of intravenously administered bFGF and the effect of heparin. Growth Factors. 1:157-164.

55. Cordeiro, P. G., B. R. Seckel, S. A. Lipton, P. A. D'Amore, J. Wagner, and R. Madison. 1989. Acidic fibroblast growth factor enhances peripheral nerve regeneration in vivo. Plast. Reconstr. Surg. 83:1013-1019. 